\title{
Visible-Light-Driven $\mathrm{C}-\mathrm{N}$ Bonds Formation by a Hexa-Nickel \\ Cluster Substituted Polyoxometalate-Based Photocatalyst
}

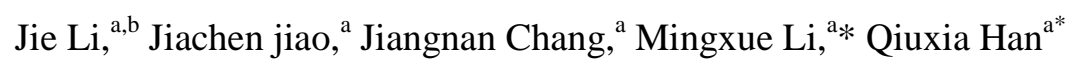

${ }^{a}$ Henan Key Laboratory of Polyoxometalates, Institute of Molecular and Crystal Engineering, College of Chemistry and Chemical Engineering, Henan University, Kaifeng 475004, PR China

${ }^{b}$ School of Chemistry \& Chemical Engineering, Zhoukou Normal University, Zhoukou 466001, PR China

Corresponding Author: limingxue @henu.edu.cn; hdhqx@ @henu.edu.cn

1. Materials and characterizations

All chemicals were purchased commercial without further purification. $\mathrm{Na}_{10}\left[\alpha-\mathrm{SiW}_{9} \mathrm{O}_{34}\right] \cdot \mathrm{H}_{2} \mathrm{O}$ and DPNDI were prepared according to a literature procedure ${ }^{1,2}$. Elemental analysis of $\mathrm{C}, \mathrm{H}$ and $\mathrm{N}$ was taken on a Perkin-Elmer 2400-II CHNS/O analyzer. Inductively coupled plasma (ICP) spectra of $\mathrm{Si}, \mathrm{Ni}$ and $\mathrm{W}$ elements were conducted on a Perkin-Elmer Optima 2000 ICP-OES spectrometer. Powder X-ray diffraction (PXRD) patterns were conducted using a Bruker AXS D8 Advance diffractometer instrument with $\mathrm{Cu} \mathrm{K} \alpha$ radiation $(\lambda=1.54056 \AA)$ at $293 \mathrm{~K}$. Fourier transform infrared spectroscopy (FTIR) spectra were recorded on a Bruker VERTEX 70 using KBr tables in the range of 500-4000 $\mathrm{cm}^{-1}$. UV-vis spectra were collected on a UH4150 spectrometer. Thermogravimetric analyses (TGA) was conducted on a NETZSCH STA449F5/QMS 403D instrument from 35 to $1000^{\circ} \mathrm{C}$ under $\mathrm{N}_{2}$ at a rate of $10^{\circ} \mathrm{C} / \mathrm{min}$. X-ray photoelectron spectra (XPS) were obtained with $\mathrm{Al} \mathrm{Ka}(1486.6 \mathrm{eV})$ on a Thermo ESCALAB 250XI photoelectron spectrometer. The magnetic property was explored by a Lakeshore 7300 . The electrical conductivity data were obtained on a 1260 A solarton analytical. Mott-Schottky plots were conducted on an electrochemical workstation (CHI 760E). The scanning electron microscopy (SEM) and mapping spectra of sample were performed on a (JSM-7610F). Gas chromatography (GC) was performed with an Agilent Technologies 7890B-MSD 59778 instrument using $99.99 \% \mathrm{~N}_{2}$ as a carrier gas. ${ }^{1} \mathrm{H}$ NMR was performed on an AVANCE III HD $400 \mathrm{MHz}$ spectrometer with chemical shifts reported as ppm $(\delta)$ and coupling constants $(J)$ are reported in $\mathrm{Hz}$ (in $\mathrm{CDCl}_{3}$, TMS as internal standard). Photoluminescence properties were explored on an EDINBURGH FLS 980 fluorescence spectrophotometer equipped with a $450 \mathrm{~W}$ xenon lamp.

\section{X-ray crystallography}

The data of $\mathrm{SiW}_{9} \mathrm{Ni}_{6}$-DPNDI was collecteded on a Bruker D8 VENTURE PHOTON II CCD diffractometer with graphite-monochromated Mo K $\alpha(\lambda=0.71073 \AA)$ at $150(2) \mathrm{K}$ using SAINT and SMART programs. Multi-scan absorption correction and Routine Lorentz polarization were used for the intensity data. The structure was determined by direct procedures with the SHELX program package and refined with the ShelXL-2018/3 package using least squares minimisation. All the atoms were refined anisotropically in the last refinement cycle. $\mathrm{H}$ atoms were fixed in calculated positions and then refined using a riding model. All of them are directly included in the molecular formula. The CCDC number of $\mathrm{SiW}_{9} \mathrm{Ni}_{6}-\mathrm{DPND}$ is 2053687. Crystallographic data and structure refinements for $\mathrm{SiW}_{9} \mathrm{Ni}_{6}$-DPND are listed in Table $\mathrm{S} 1$. 
Table S1. Crystallographic data and structure refinements for $\mathrm{SiW}_{9} \mathrm{Ni}_{6}$-DPNDI.

\begin{tabular}{|c|c|}
\hline & $\mathrm{SiW}_{9} \mathrm{Ni}_{6}$-DPNDI \\
\hline Empirical formula & $\mathrm{C}_{26} \mathrm{H}_{40} \mathrm{~N}_{4} \mathrm{Ni}_{6} \mathrm{O}_{51} \mathrm{SiW}_{9}$ \\
\hline Formular weight & 3259.40 \\
\hline Crystal system & Monoclinic \\
\hline Space group & $C 2 / c$ \\
\hline$a$ & $38.082(8)$ \\
\hline$b$ & $16.806(3)$ \\
\hline$c$ & $29.039(6)$ \\
\hline$\beta$ & $109.501(7)$ \\
\hline Volume/[ $\left[\AA^{3}\right]$ & $17519(6)$ \\
\hline$Z$ & 8 \\
\hline Calculated density $/\left[\mathrm{g} \cdot \mathrm{cm}^{-3}\right]$ & 2.455 \\
\hline Absorption coefficient $/\left[\mathrm{mm}^{-1}\right]$ & 13.114 \\
\hline $\mathrm{F}(000)$ & 11648 \\
\hline $2 \theta$ range for data collection & 4.274 to $50.198^{\circ}$ \\
\hline Limiting indices & $-45 \leq \mathrm{h} \leq 45,-19 \leq \mathrm{k} \leq 19,-34 \leq \mathrm{l} \leq 33$ \\
\hline Reflections collected/unique & $54937 / 15383\left[R_{\text {int }}=0.0716, R_{\text {sigma }}=0.0722\right]$ \\
\hline Data/restraints/parameters & $15383 / 25 / 850$ \\
\hline Goodness-of-fit on $\mathrm{F}^{2}$ & 1.123 \\
\hline Final $R$ indices $[I>2 \sigma(I)]^{a}$ & $R_{1}=0.0622, w R_{2}=0.1791$ \\
\hline $\mathrm{R}$ indices (all data) ${ }^{\mathrm{b}}$ & $R_{1}=0.0778, w R_{2}=0.1917$ \\
\hline Largest diff. peak and hole/[e $\left.\cdot \AA^{-3}\right]$ & 2.98 and -3.39 \\
\hline
\end{tabular}




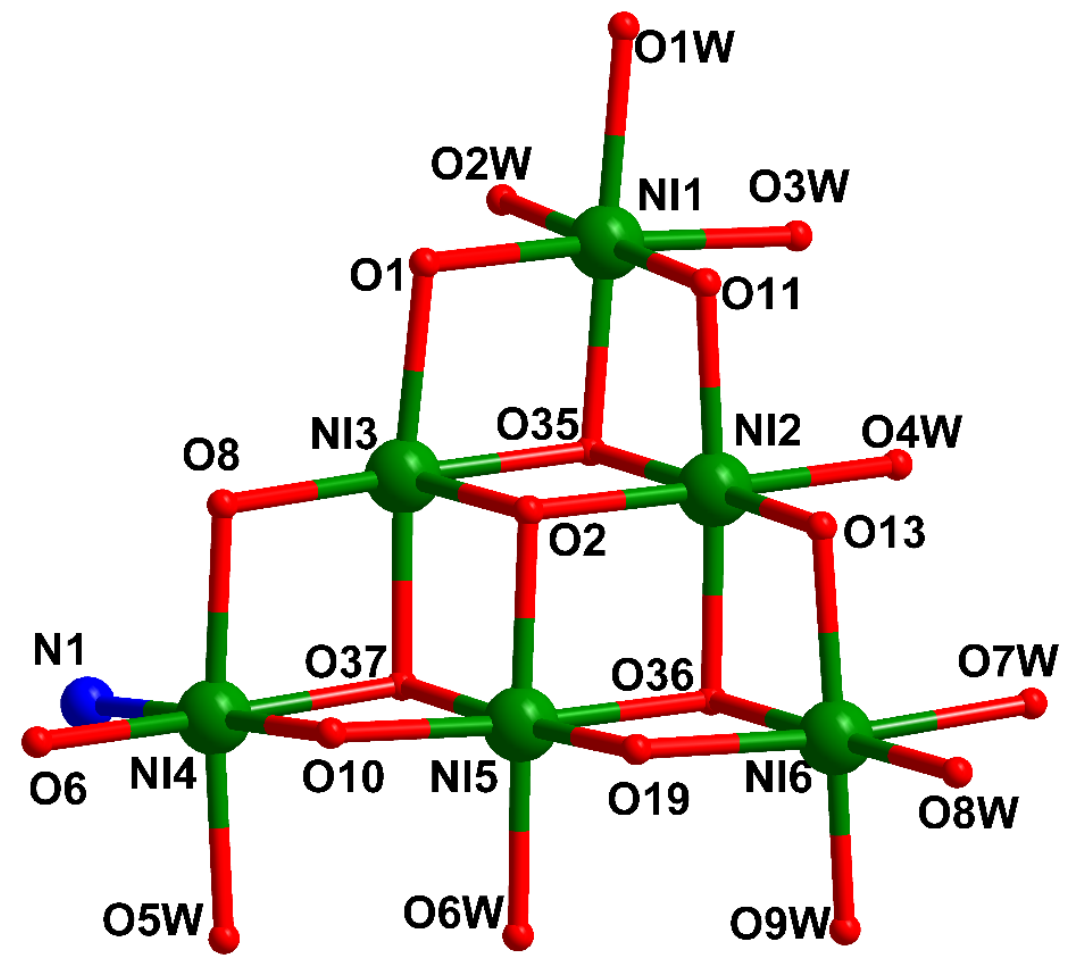

Figure S1. The coordination mode of different $\mathrm{Ni}^{2+}$ ions of $\mathrm{SiW}_{9} \mathrm{Ni}_{6}$-DPNDI. 
Table $\mathrm{S} 2$. BVS results of $\mathrm{W}, \mathrm{Si}, \mathrm{Ni}$ and $\mathrm{O}$ atoms.

\begin{tabular}{cccccc}
\hline Atom & $\begin{array}{c}\text { Bond } \\
\text { valence sum }\end{array}$ & Atom & $\begin{array}{c}\text { Bond } \\
\text { valence sum }\end{array}$ & Atom & $\begin{array}{c}\text { Bond } \\
\text { valence sum }\end{array}$ \\
\hline W1 & 6.163 & W2 & 5.969 & W3 & 5.872 \\
W4 & 5.943 & W5 & 6.077 & W6 & 6.148 \\
W7 & 5.828 & W8 & 5.981 & W9 & 6.094 \\
Si1 & 3.672 & Ni1 & 1.857 & Ni2 & 1.953 \\
Ni3 & 1.519 & Ni4 & 1.645 & Ni5 & 1.923 \\
Ni6 & 1.877 & O1 & 1.941 & O2 & 1.825 \\
O3 & 1.936 & O4 & 1.793 & O5 & 1.777 \\
O6 & 1.906 & O7 & 1.769 & O8 & 1.968 \\
O9 & 1.798 & O10 & 1.968 & O11 & 1.910 \\
O12 & 1.950 & O13 & 1.866 & O14 & 1.985 \\
O15 & 1.596 & O16 & 1.790 & O17 & 1.932 \\
O18 & 1.456 & O19 & 1.808 & O20 & 1.890 \\
O21 & 1.886 & O22 & 1.778 & O23 & 1.874 \\
O24 & 1.958 & O25 & 1.968 & O26 & 1.788 \\
O27 & 1.671 & O28 & 1.802 & O29 & 1.818 \\
O30 & 1.888 & O31 & 1.743 & O32 & 1.605 \\
O33 & 1.600 & O34 & 1.622 & O35 & 1.036 \\
O36 & 0.825 & O37 & 1.104 & O41 & 1.500 \\
O42 & 1.541 & O43 & 1.208 & O44 & 1.500 \\
\hline
\end{tabular}




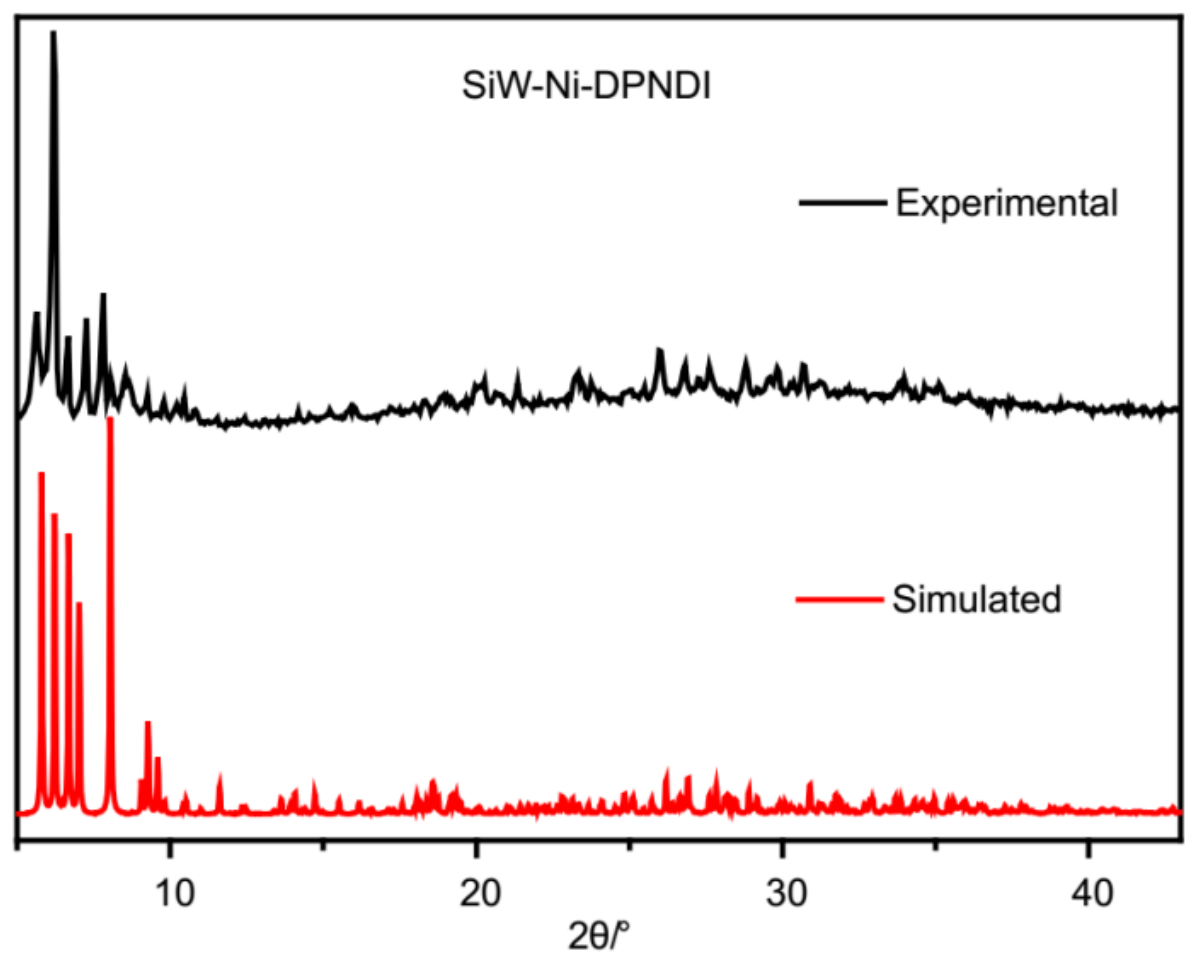

Figure S2. PXRD patterns of $\mathrm{SiW}_{9} \mathrm{Ni}_{6}$-DPNDI.

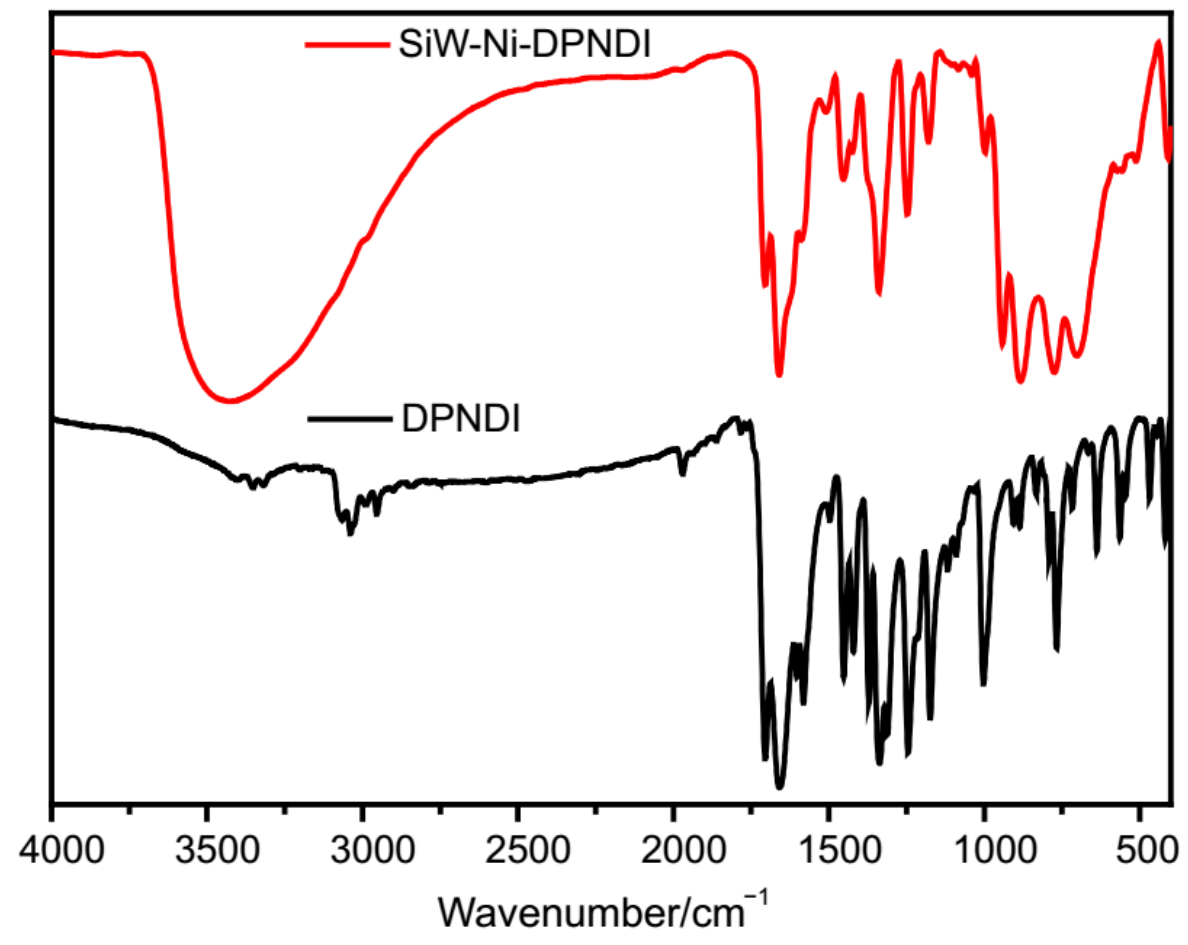

Figure S3. FTIR spectra of $\mathrm{SiW}_{9} \mathrm{Ni}_{6}$-DPNDI and DPNDI ligand. 


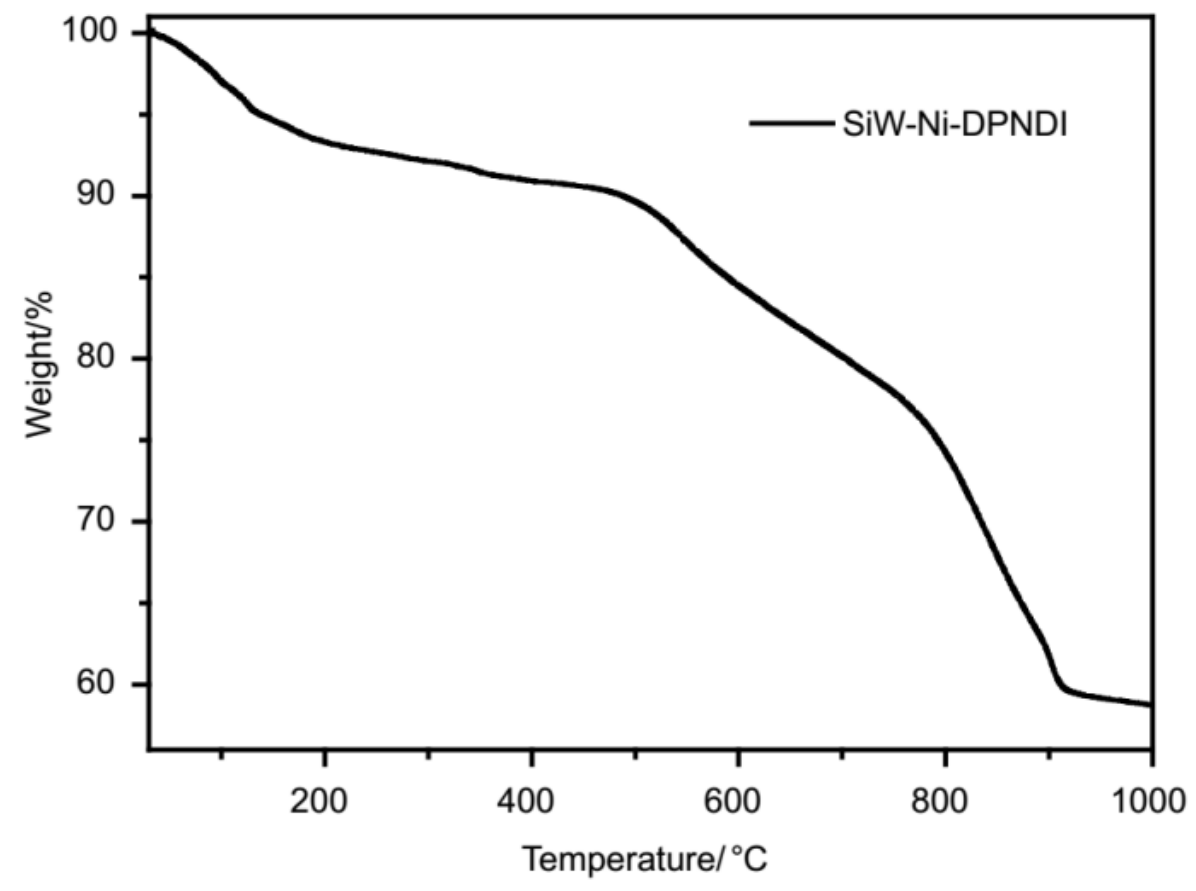

Figure S4. TGA spectra of $\mathrm{SiW}_{9} \mathrm{Ni}_{6}$-DPNDI. 
The proton conductivity $(\sigma)$ of $\mathrm{SiW}_{9} \mathrm{Ni}_{6}$-DPNDI was explored by alternating current impedance spectroscopy utilizing a compressed pellet of powered crystalline sample over the temperature range of 298-358 K at 98\% relative humidity. All the Nyquist plots were shown in Figure S5a. The $\mathrm{Z}^{\prime}$-axis intercept value is approximately considered as the resistance of the $\mathrm{SiW}_{9} \mathrm{Ni}_{6}-\mathrm{DPNDI}^{3}$, which shows a decreasing with the temperature increasing. The results indicated that the thermal activation was beneficial for the proton conductivity increasing. The proton conductivities of $\mathrm{SiW}_{9} \mathrm{Ni}_{6}$-DPNDI at $298,308,318,328,338,348$, and $358 \mathrm{~K}$ were $6.52 \times 10^{-6} \mathrm{~S} \mathrm{~cm}^{-1}, 1.98 \times 10^{-5} \mathrm{~S}$ $\mathrm{cm}^{-1}, 3.62 \times 10^{-5} \mathrm{~S} \mathrm{~cm}^{-1}, 4.76 \times 10^{-5} \mathrm{~S} \mathrm{~cm}^{-1}, 6.23 \times 10^{-5} \mathrm{~S} \mathrm{~cm}^{-1}, 8.60 \times 10^{-5} \mathrm{~S} \mathrm{~cm}^{-1}$, and $1.18 \times 10^{-4} \mathrm{~S}$ $\mathrm{cm}^{-1}$, respectively. Although the highest proton conductivity of $\mathrm{SiW}_{9} \mathrm{Ni}_{6}$-DPNDI was not remarkable, it is still higher than many materials. The plot of $\ln (\sigma \mathrm{T})$ vs $1000 \mathrm{~T}^{-1}$ was approximate liner utilizing the Arrhenius equation, suggesting the value of Ea was $0.43 \mathrm{~V}$ (Figure S5b). According to the previous literature, the proton-conducting behavior in $\mathrm{SiW}_{9} \mathrm{Ni}_{6}$-DPNDI was through the Vehicle mechanism ${ }^{4}$. The high proton conductivity of $\mathrm{SiW}_{9} \mathrm{Ni}_{6}$-DPNDI is ascribed to the formation of hydrogen-bonding network among the terminal oxygen atom, nitrogen atoms, counter cations $\mathrm{H}^{+}$, and water molecules ${ }^{5}$.
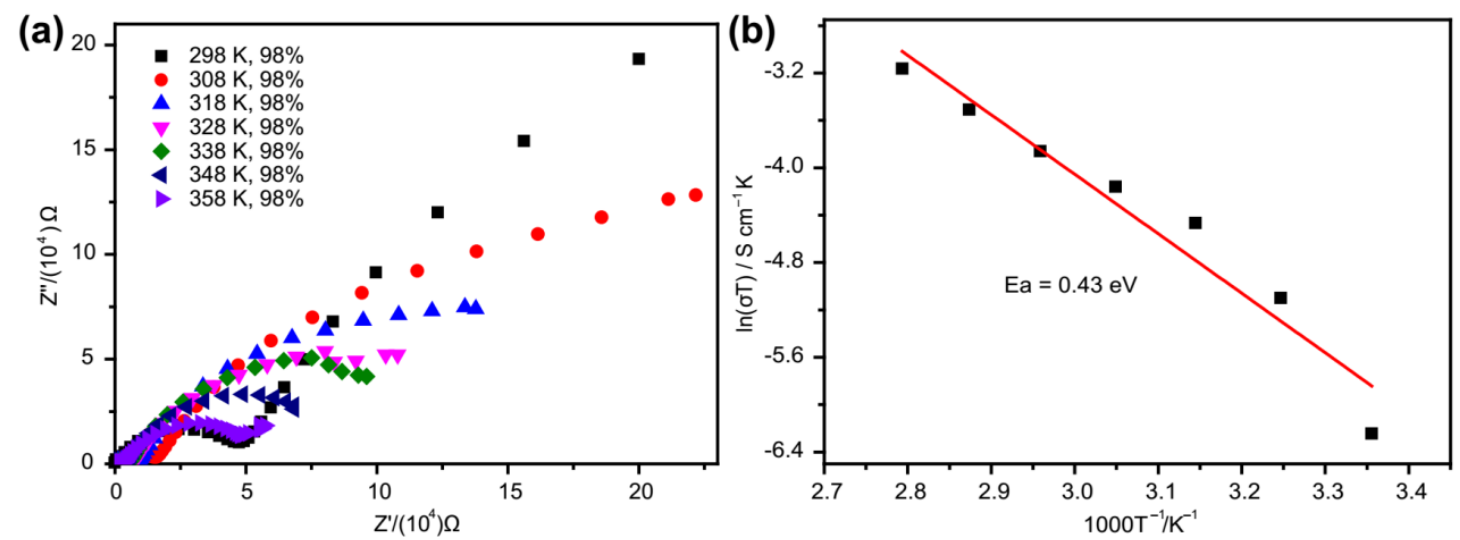

Figure S5. (a) Nyquist curves of $\mathrm{SiW}_{9} \mathrm{Ni}_{6}$-DPNDI at different temperature. (b) Arrhenius plot of conductivity. 


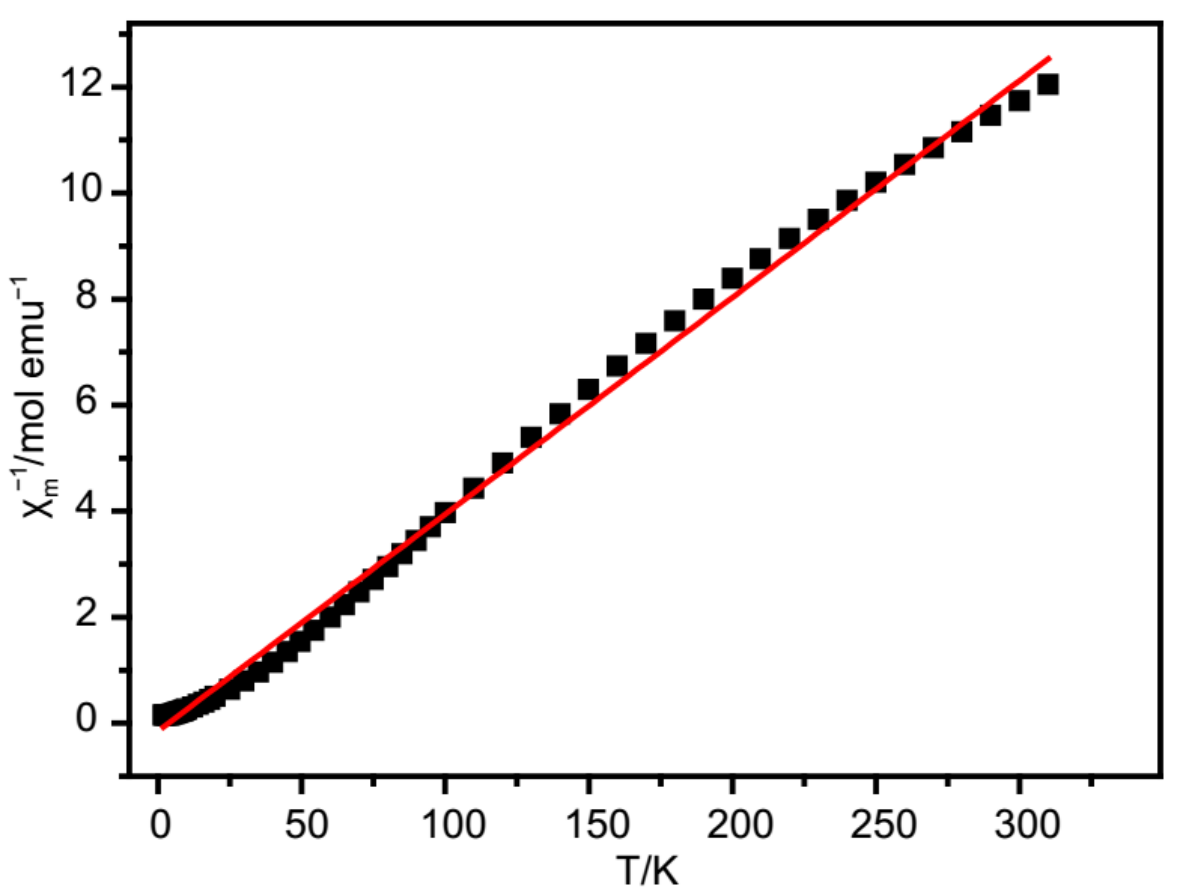

Figure S6. The $\chi_{m}{ }^{-1}$ vs. $\mathrm{T}$ curve of $\mathrm{SiW}_{9} \mathrm{Ni}_{6}$-DPNDI.

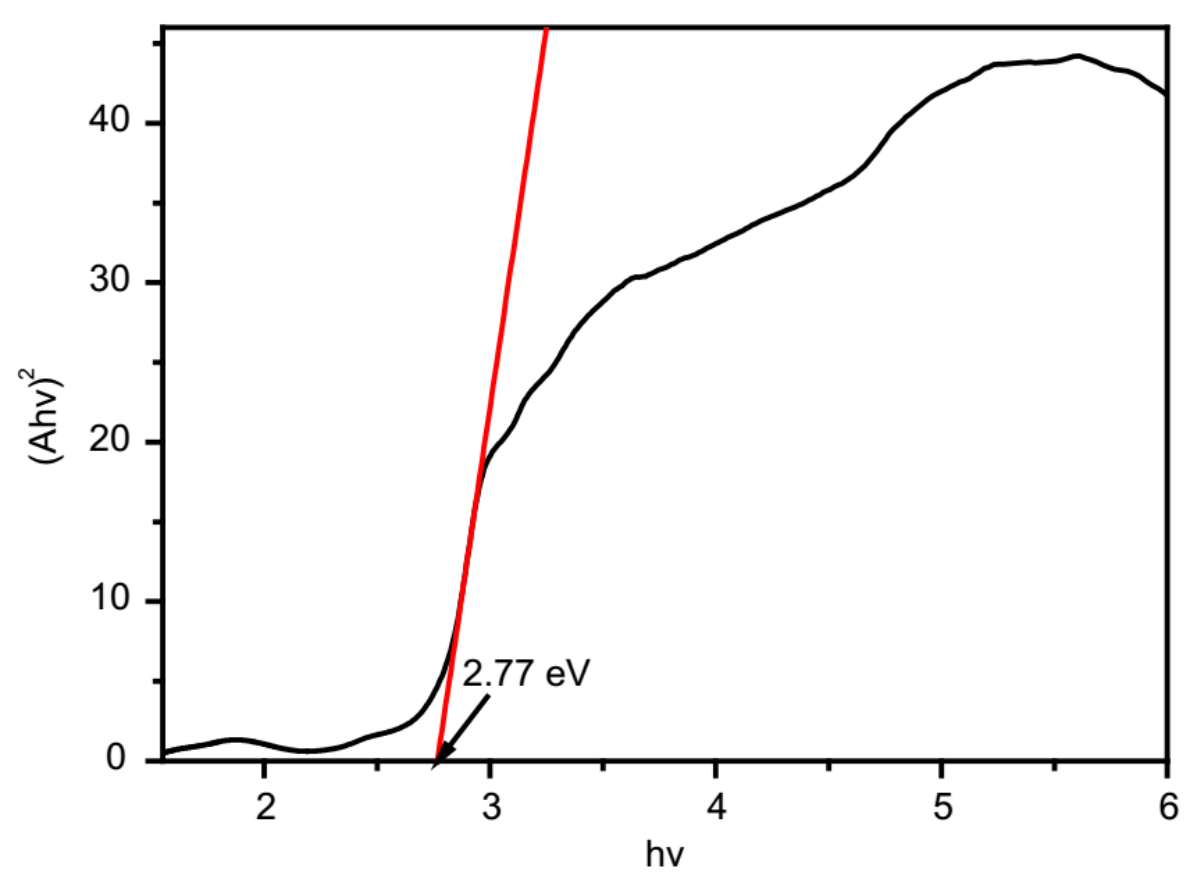

Figure S7. Tauc plot of $\mathrm{SiW}_{9} \mathrm{Ni}_{6}$-DPNDI. 


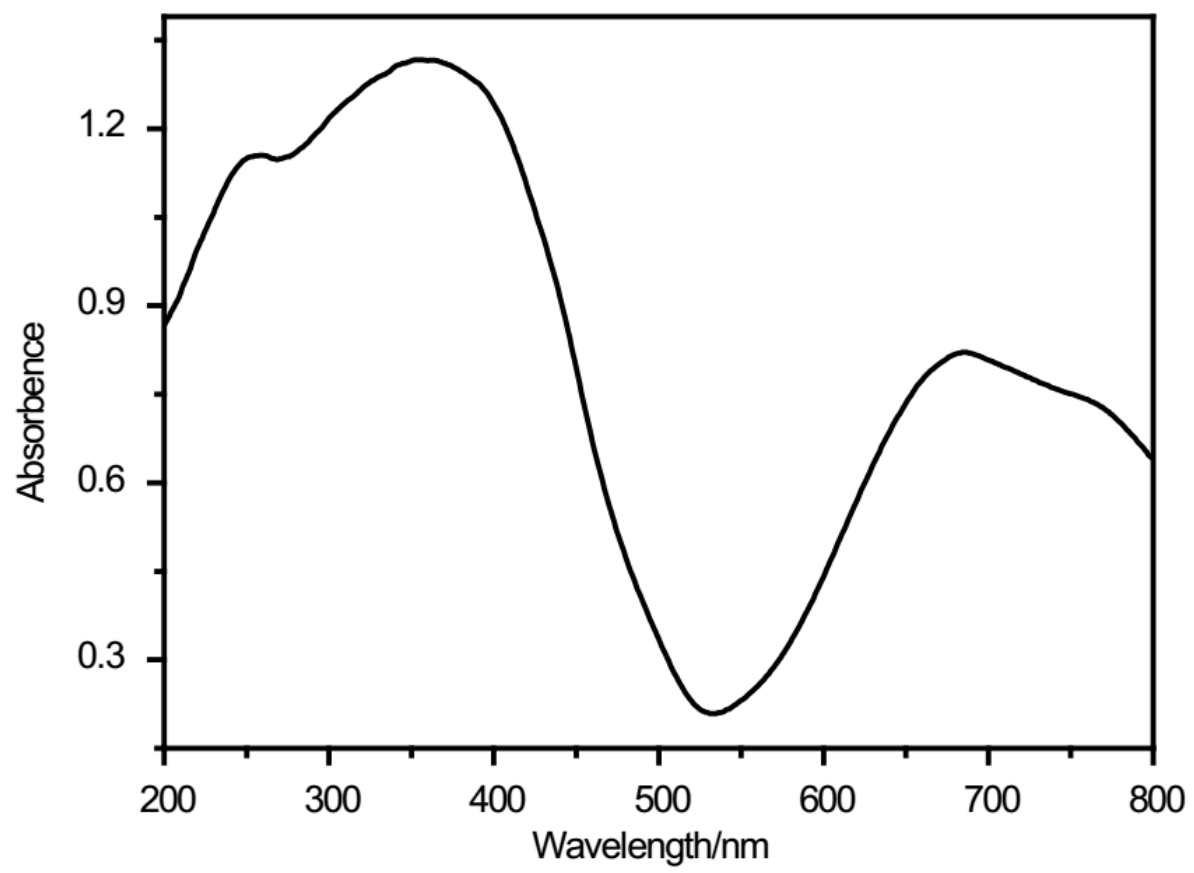

Figure S8. The UV-vis spectra of $\mathrm{SiW}_{9} \mathrm{Ni}_{6}$.

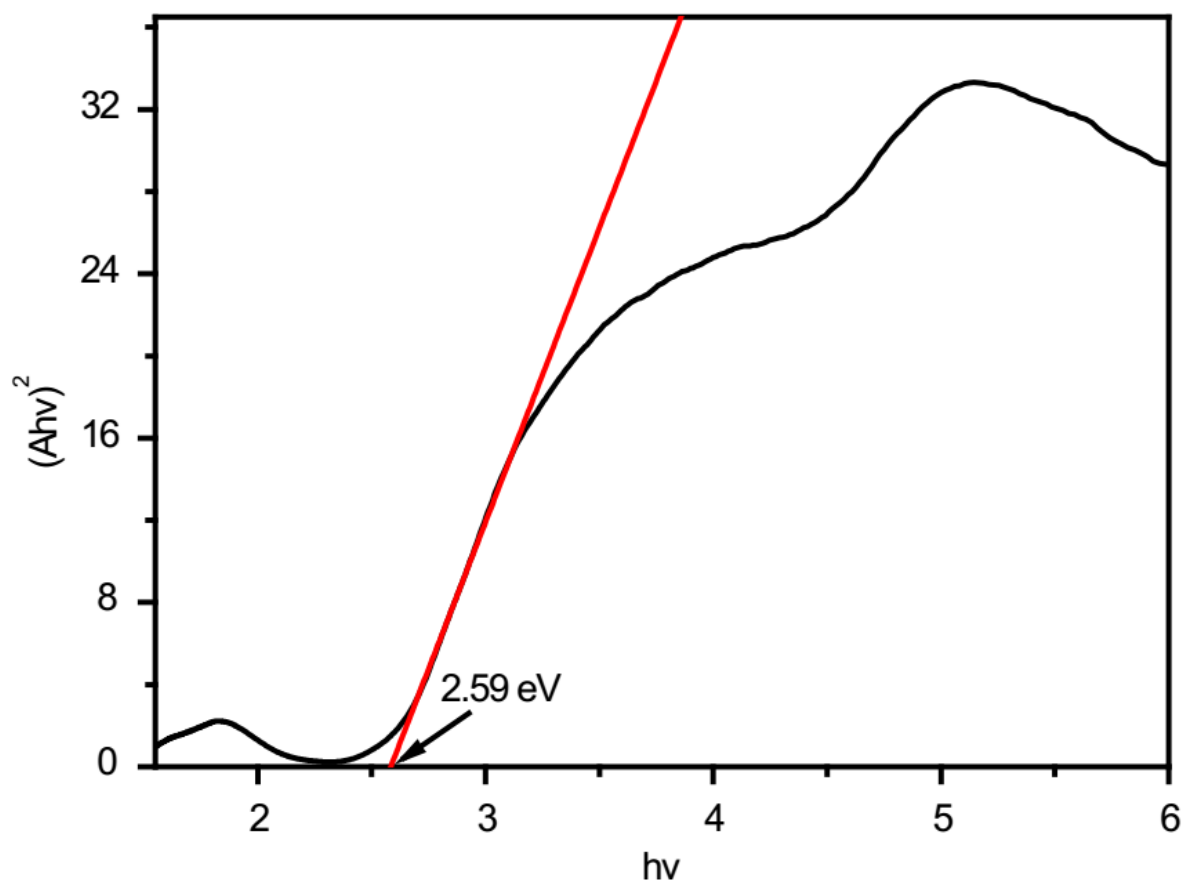

Figure S9. Tauc plot of $\mathrm{SiW}_{9} \mathrm{Ni}_{6}$. 


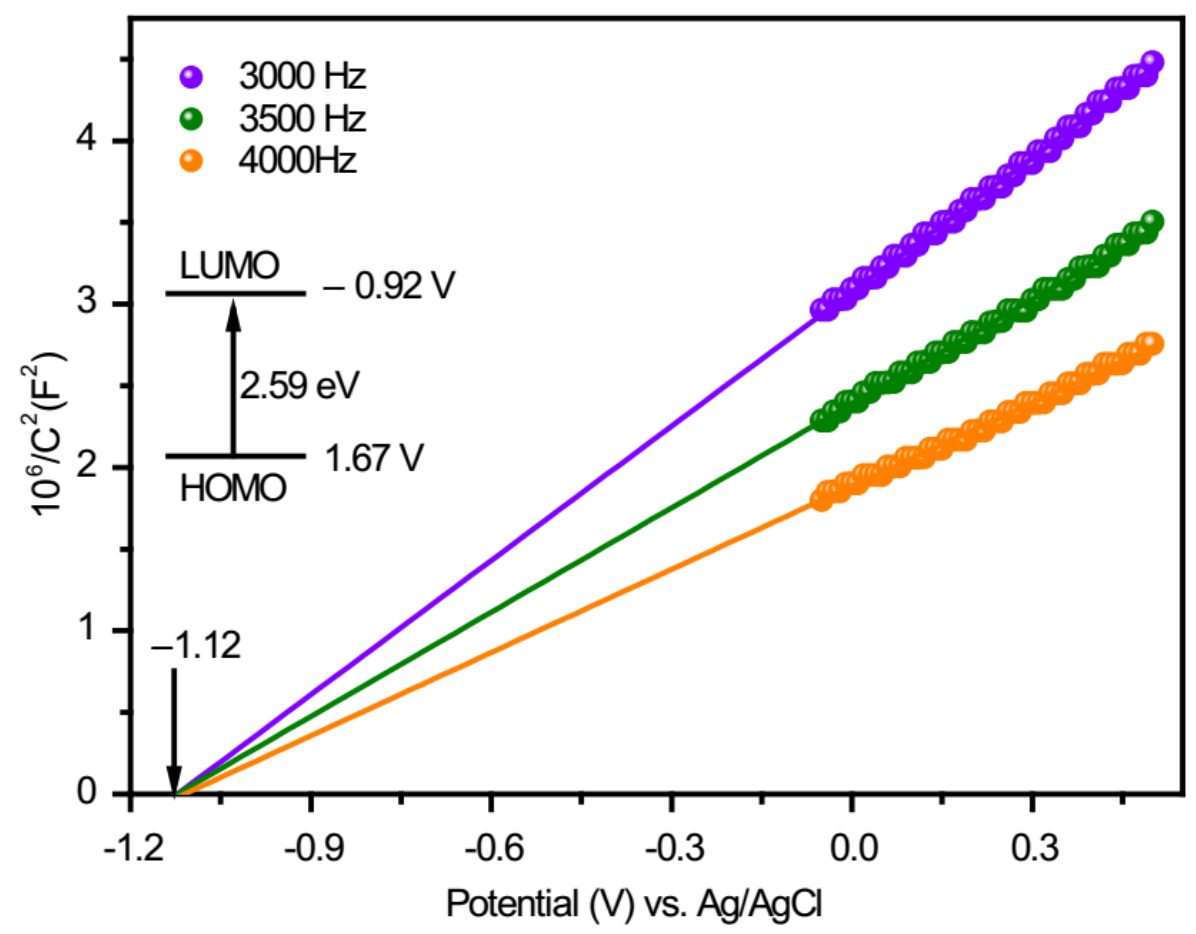

Figure S10. The HUMO and LUMO analysis of $\mathrm{SiW}_{9} \mathrm{Ni}_{6}$.
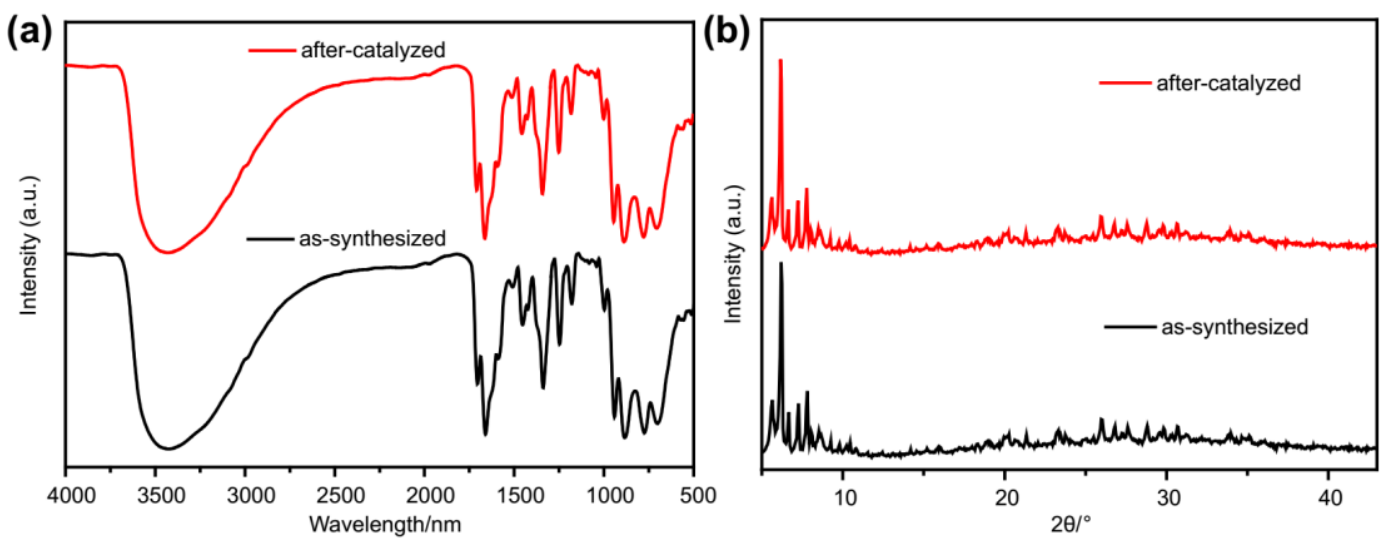

Figure S11. The as-synthesized and after-adsorption IR and PXRD spectra of $\mathrm{SiW}_{9} \mathrm{Ni}_{6}-$ DPNDI. 


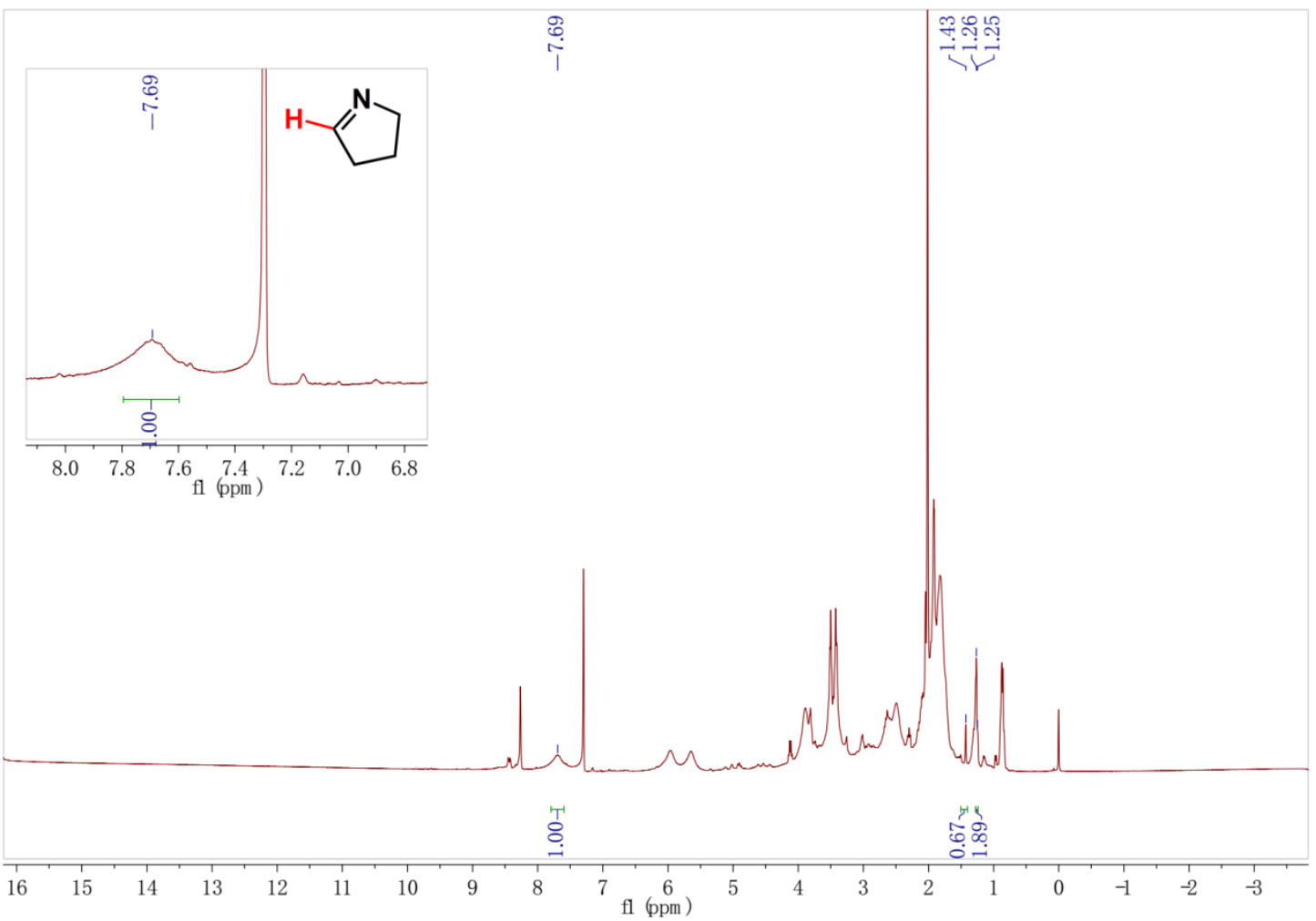

${ }^{1} \mathrm{H}$ NMR (400 MHz, CDCl3) $\delta 7.69$ (s, 1H), $1.43(\mathrm{~s}, 1 \mathrm{H}), 1.26(\mathrm{~d}, \mathrm{~J}=6.7 \mathrm{~Hz}, 2 \mathrm{H})$.

Figure S12. The intermediate of the reaction was confirmed by ${ }^{1} \mathrm{H}$ NMR. 
(4-bromophenyl)(pyrrolidin-1-yl)methanone<smiles>O=C(c1ccc(Br)cc1)N1CCCC1</smiles>

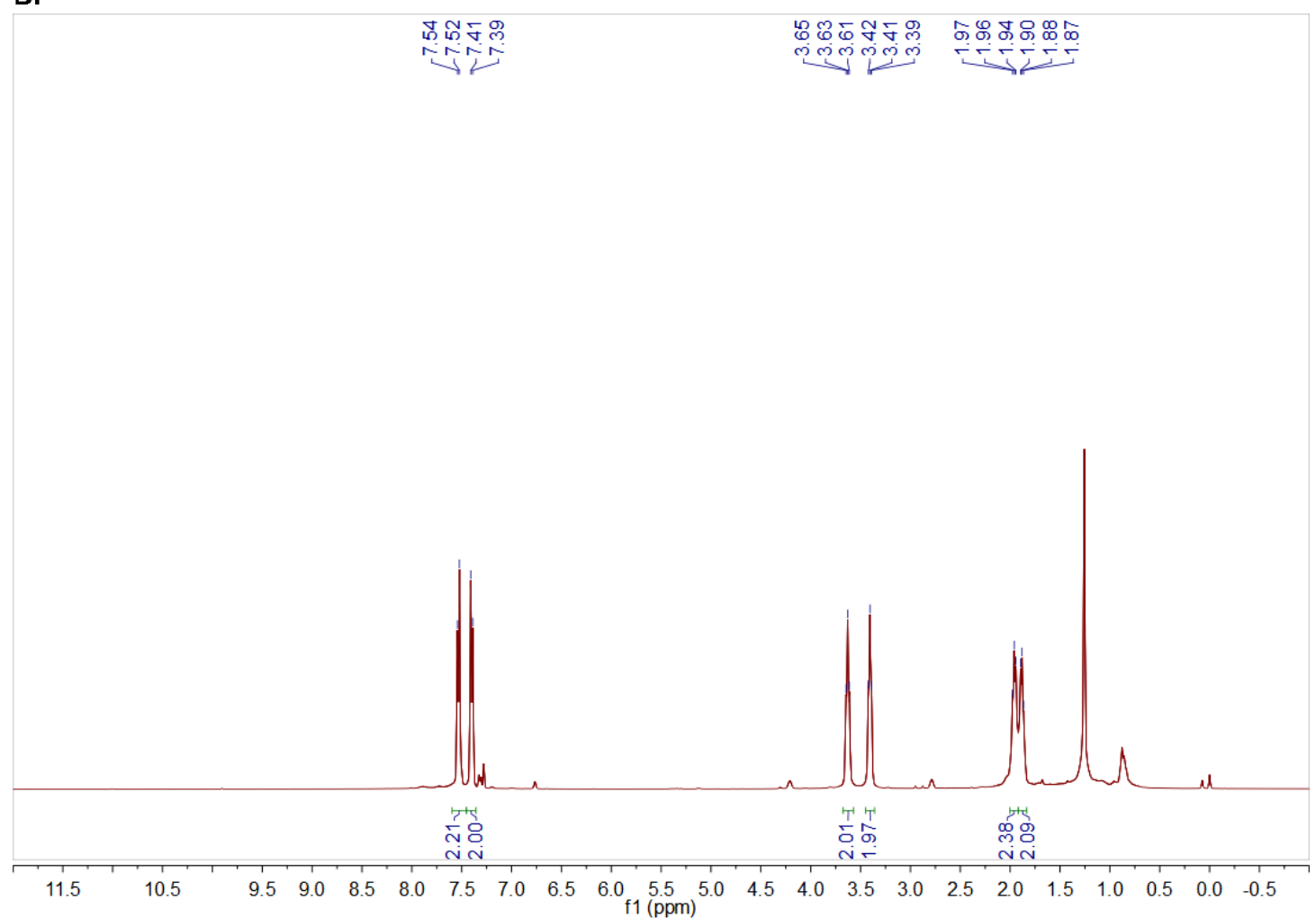

${ }^{1} \mathrm{H}$ NMR $\left(400 \mathrm{MHz}, \mathrm{CDCl}_{3}\right) \delta 7.53(\mathrm{~d}, J=8.0 \mathrm{~Hz}, 2 \mathrm{H}), 7.40(\mathrm{~d}, J=8.1 \mathrm{~Hz}, 2 \mathrm{H}), 3.63(\mathrm{t}, J=6.4$ $\mathrm{Hz}, 2 \mathrm{H}), 3.41(\mathrm{t}, J=6.0 \mathrm{~Hz}, 2 \mathrm{H}), 2.01-1.92(\mathrm{~m}, 2 \mathrm{H}), 1.92-1.82(\mathrm{~m}, 2 \mathrm{H})$. 
(4-chlorophenyl)(pyrrolidin-1-yl)methanone<smiles>O=C(c1ccc(Cl)cc1)N1CCCC1</smiles>

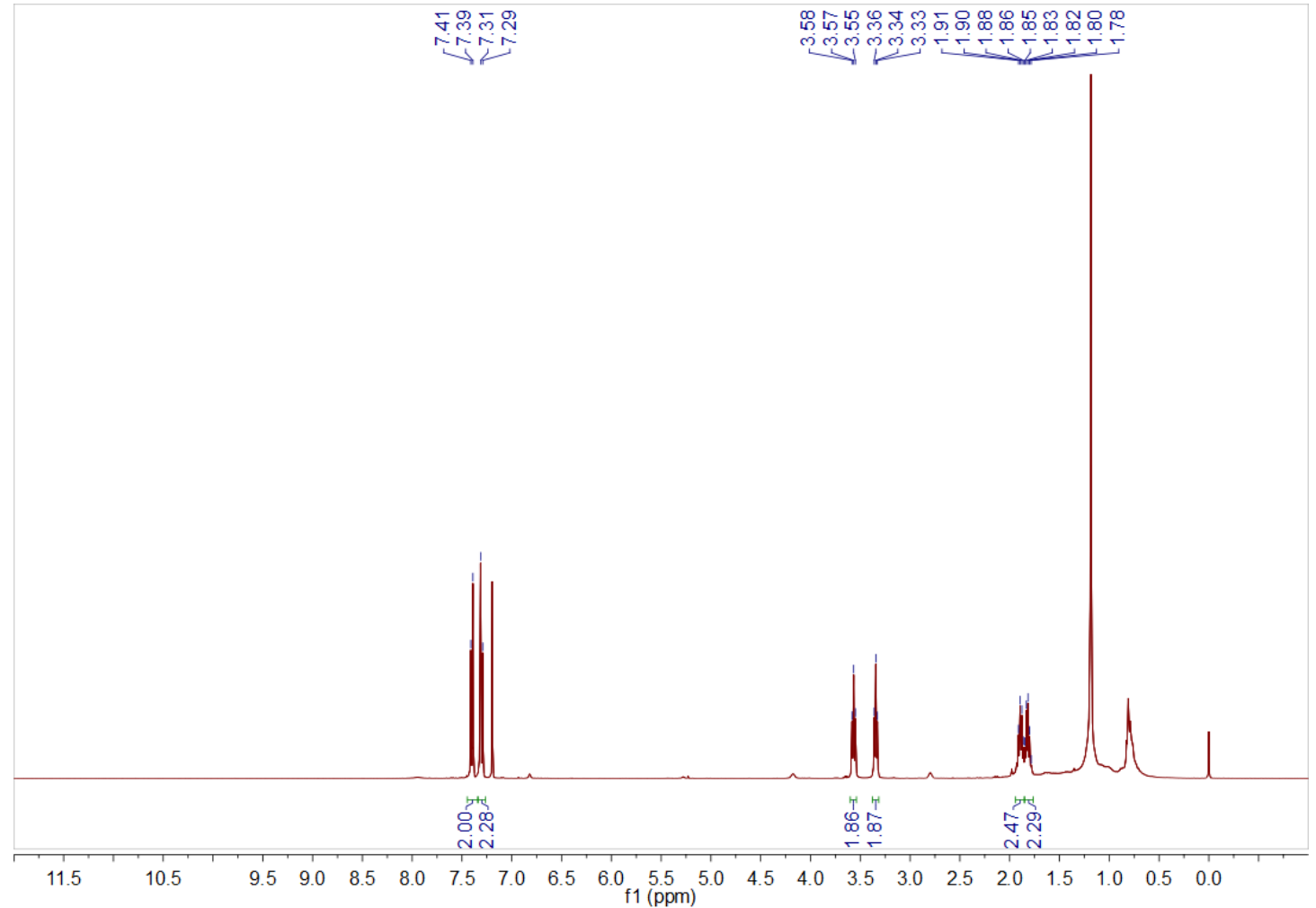

${ }^{1} \mathrm{H}$ NMR $\left(400 \mathrm{MHz}, \mathrm{CDCl}_{3}\right) \delta 7.40(\mathrm{~d}, J=8.5 \mathrm{~Hz}, 2 \mathrm{H}), 7.30(\mathrm{~d}, J=8.5 \mathrm{~Hz}, 2 \mathrm{H}), 3.57(\mathrm{t}, J=6.9$ $\mathrm{Hz}, 2 \mathrm{H}), 3.34$ (t, $J=6.5 \mathrm{~Hz}, 2 \mathrm{H}), 1.90(\mathrm{~m}, 2 \mathrm{H}), 1.85-1.76(\mathrm{~m}, 2 \mathrm{H})$. 


\section{(4-nitrophenyl)(pyrrolidin-1-yl)methanone}<smiles>O=C(c1ccc([N+](=O)[O-])cc1)N1CCCC1</smiles>

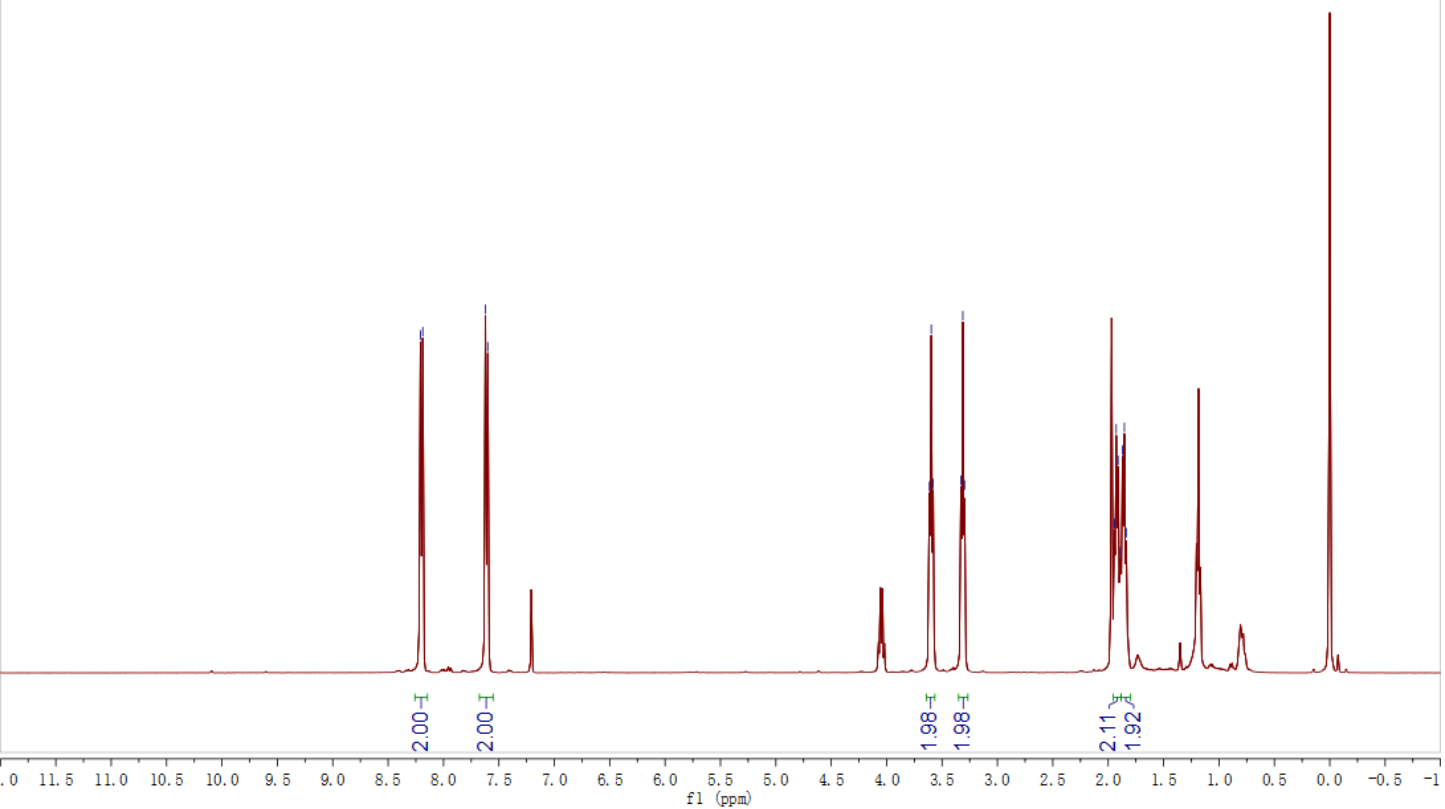

${ }^{1} \mathrm{H}$ NMR $\left(400 \mathrm{MHz}, \mathrm{CDCl}_{3}\right) \delta 8.20(\mathrm{~d}, J=8.2 \mathrm{~Hz}, 2 \mathrm{H}), 7.61(\mathrm{~d}, J=8.2 \mathrm{~Hz}, 2 \mathrm{H}), 3.60(\mathrm{t}, J=6.8$ $\mathrm{Hz}, 2 \mathrm{H}), 3.31(\mathrm{t}, J=6.4 \mathrm{~Hz}, 2 \mathrm{H}), 1.92(\mathrm{~m}, 2 \mathrm{H}), 1.89-1.80(\mathrm{~m}, 2 \mathrm{H})$. 


\section{(2-nitrophenyl)(pyrrolidin-1-yl)methanone}<smiles>O=C(c1ccccc1[N+](=O)[O-])N1CCCC1</smiles>

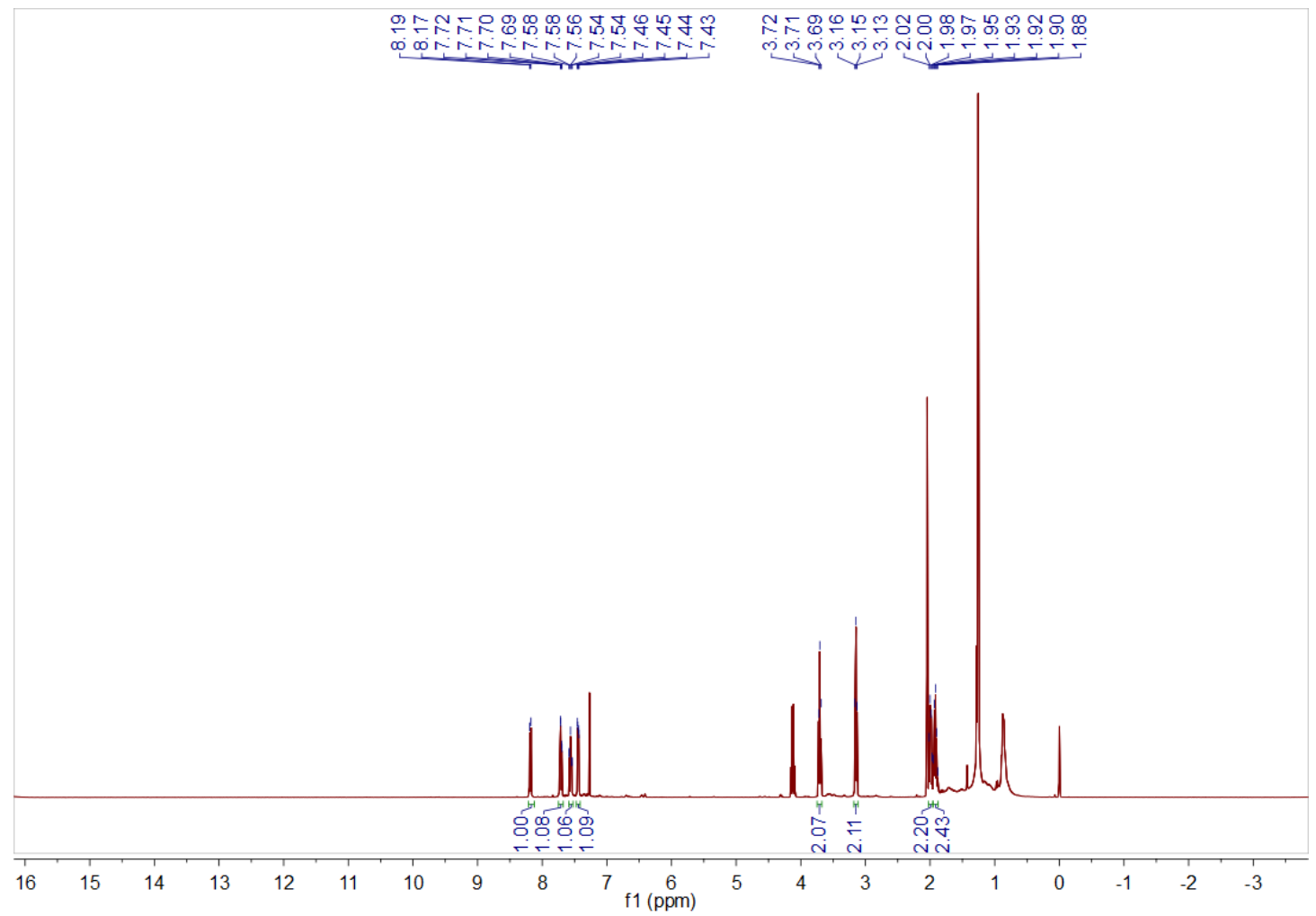

${ }^{1} \mathrm{H}$ NMR $\left(400 \mathrm{MHz}, \mathrm{CDCl}_{3}\right) \delta 8.18(\mathrm{~d}, J=8.3 \mathrm{~Hz}, 1 \mathrm{H}), 7.71(\mathrm{~m}, 1 \mathrm{H}), 7.60-7.52(\mathrm{~m}, 1 \mathrm{H}), 7.44(\mathrm{~d}$, $1 \mathrm{H}), 3.71(\mathrm{t}, J=6.9 \mathrm{~Hz}, 2 \mathrm{H}), 3.15(\mathrm{t}, J=6.7 \mathrm{~Hz}, 2 \mathrm{H}), 1.99(\mathrm{~m}, 2 \mathrm{H}), 1.95-1.87(\mathrm{~m}, 2 \mathrm{H})$. 


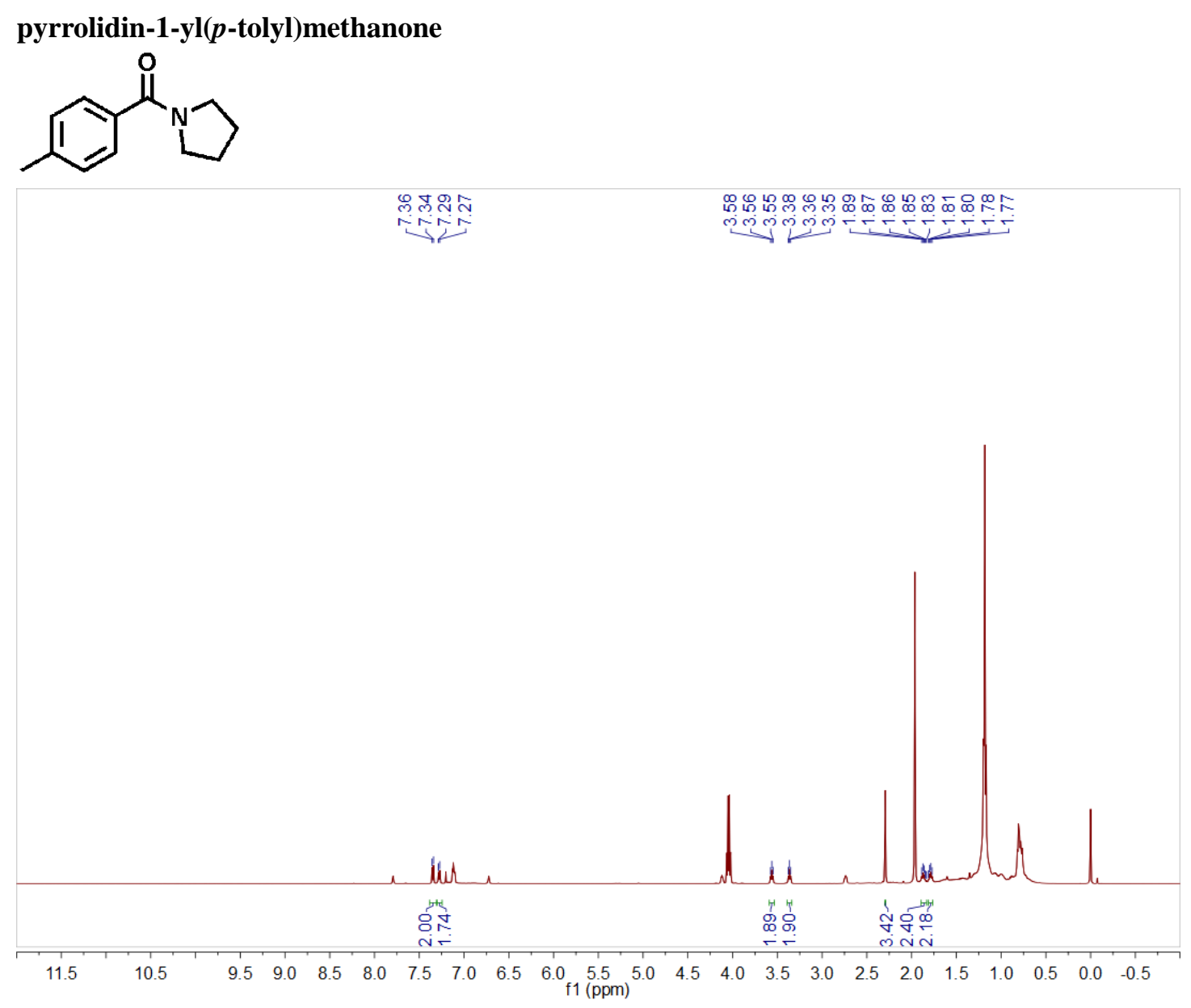

${ }^{1} \mathrm{H}$ NMR $\left(500 \mathrm{MHz}, \mathrm{CDCl}_{3}\right) \delta 7.35(\mathrm{~d}, J=7.9 \mathrm{~Hz}, 2 \mathrm{H}), 7.28(\mathrm{~d}, J=7.8 \mathrm{~Hz}, 2 \mathrm{H}), 3.56(\mathrm{t}, J=6.9$ $\mathrm{Hz}, 2 \mathrm{H}), 3.36(\mathrm{t}, J=6.6 \mathrm{~Hz}, 2 \mathrm{H}), 2.29$ (s, 3H), $1.90-1.83(\mathrm{~m}, 2 \mathrm{H}), 1.81-1.76(\mathrm{~m}, 2 \mathrm{H})$. 


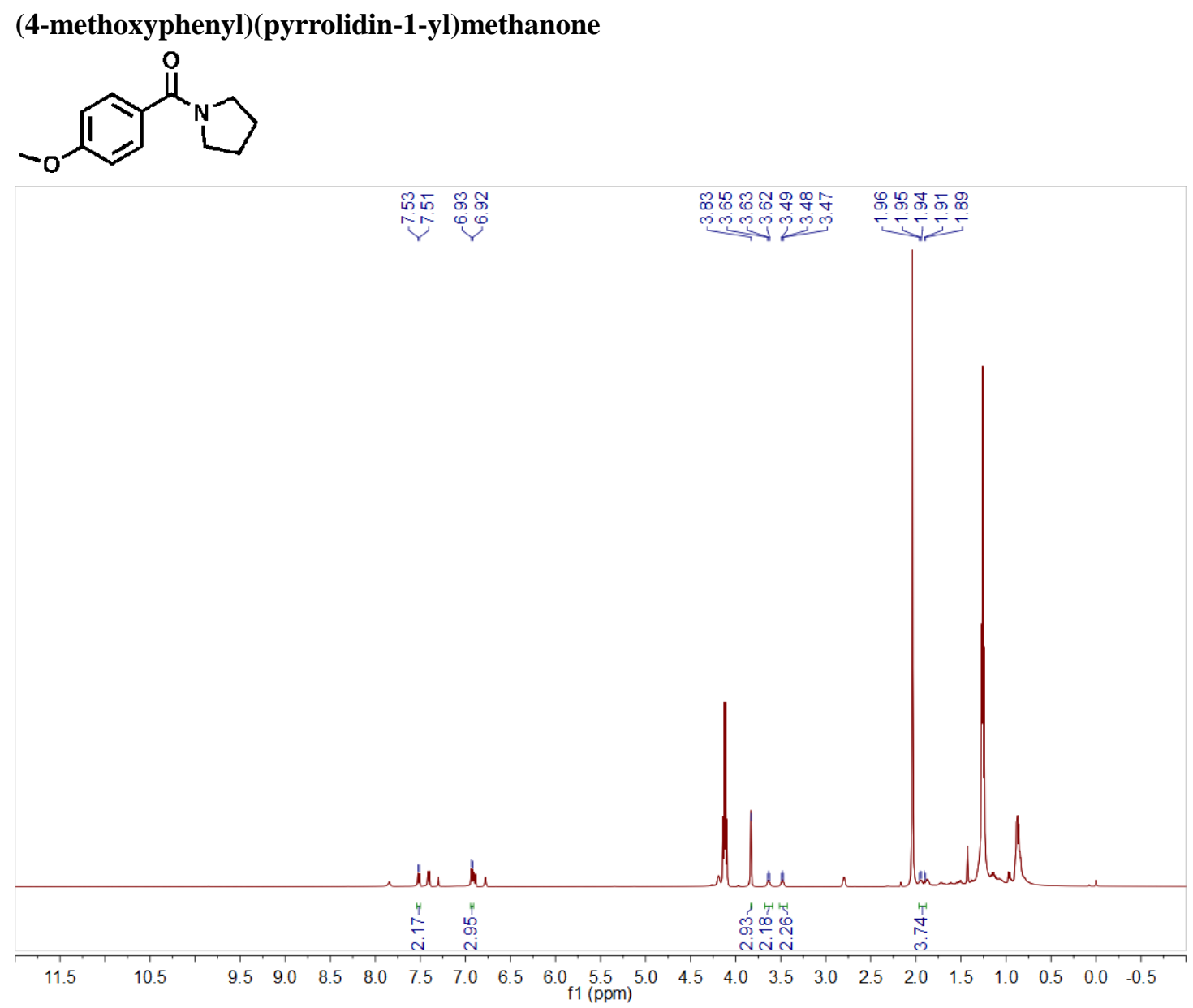

1H NMR (500 MHz, $\left.\mathrm{CDCl}_{3}\right) \delta 7.52(\mathrm{~d}, J=8.7 \mathrm{~Hz}, 2 \mathrm{H}), 6.93(\mathrm{~d}, J=8.7 \mathrm{~Hz}, 2 \mathrm{H}), 3.83(\mathrm{~s}, 3 \mathrm{H})$, $3.63(\mathrm{t}, J=6.1 \mathrm{~Hz}, 2 \mathrm{H}), 3.48(\mathrm{t}, J=5.7 \mathrm{~Hz}, 2 \mathrm{H}), 1.96-1.88(\mathrm{~m}, 4 \mathrm{H})$. 
<smiles>O=C(c1ccc([N+](=O)[O-])cc1)N1CCc2ccccc2C1</smiles>

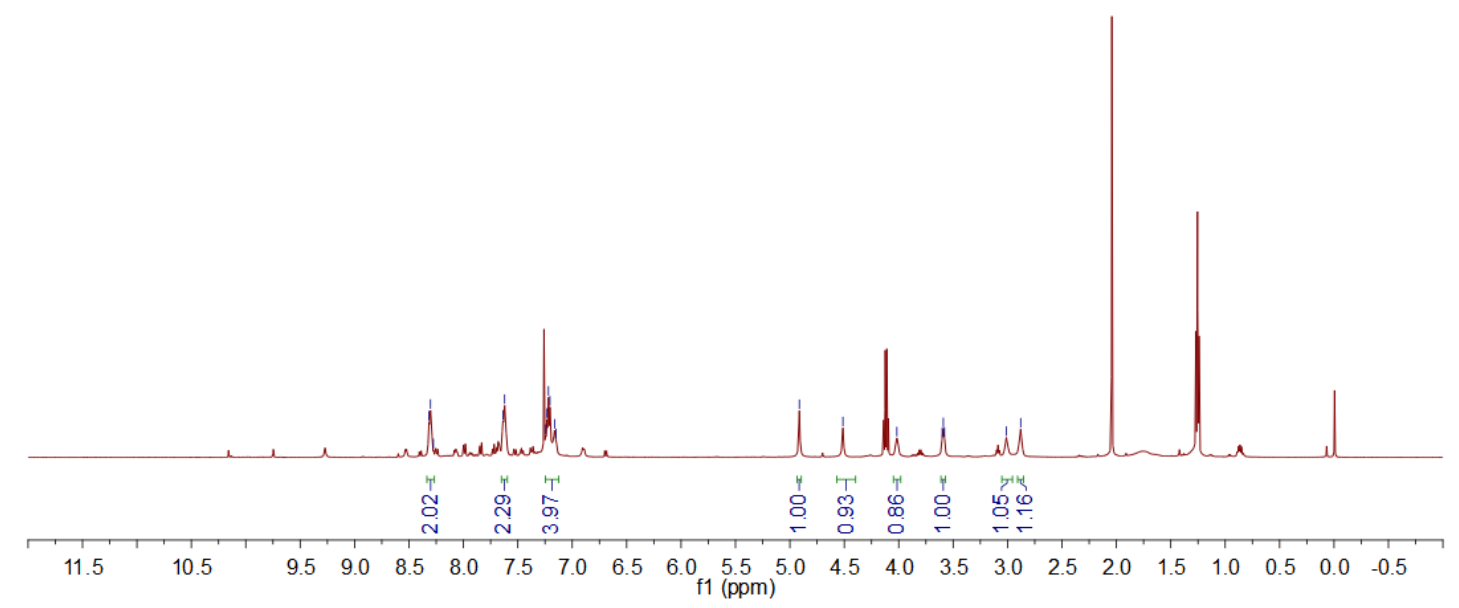

${ }^{1} \mathrm{H}$ NMR $\left(500 \mathrm{MHz}, \mathrm{CDCl}_{3}\right) \delta 8.30(\mathrm{t}, J=10.1 \mathrm{~Hz}, 2 \mathrm{H}), 7.63(\mathrm{~d}, J=7.8 \mathrm{~Hz}, 2 \mathrm{H}), 7.25-7.12(\mathrm{~m}$, $4 \mathrm{H}), 4.91(\mathrm{~s}, 1 \mathrm{H}), 4.51(\mathrm{~s}, 1 \mathrm{H}), 4.02(\mathrm{~s}, 1 \mathrm{H}), 3.59(\mathrm{t}, J=5.2 \mathrm{~Hz}, 1 \mathrm{H}), 3.01(\mathrm{~s}, 1 \mathrm{H}), 2.88(\mathrm{~s}, 1 \mathrm{H})$. 


\section{pyridin-4-yl(pyrrolidin-1-yl)methanone}<smiles>O=C(c1ccncc1)N1CCCC1</smiles>

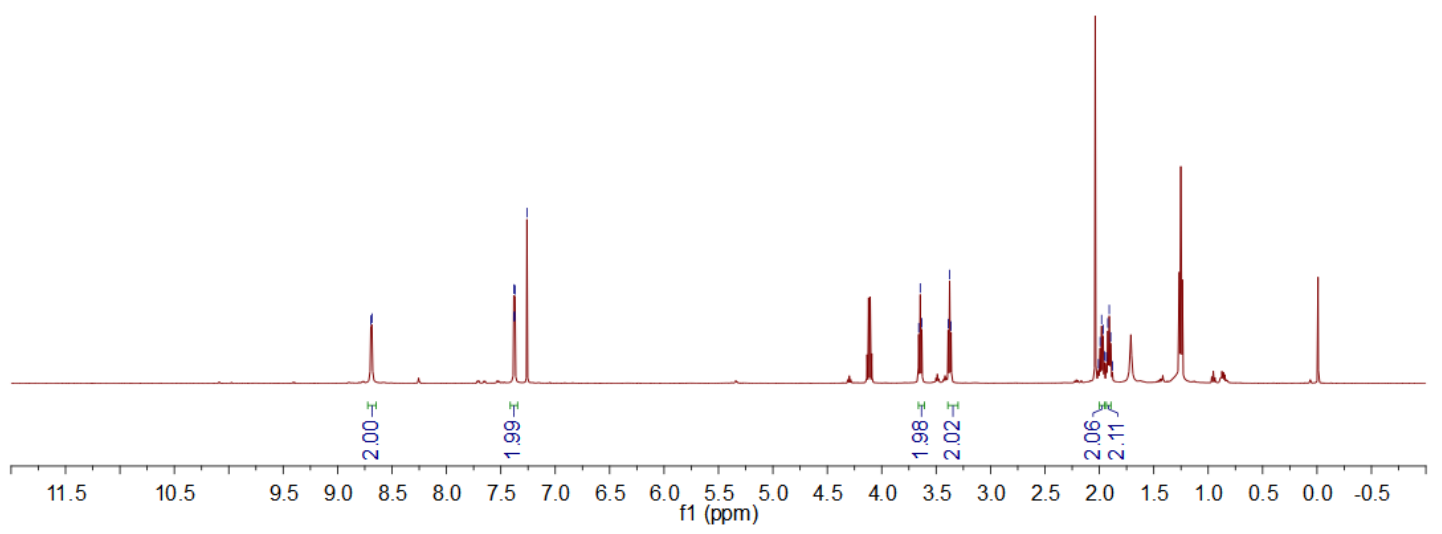

${ }^{1} \mathrm{H}$ NMR $\left(500 \mathrm{MHz}, \mathrm{CDCl}_{3}\right) \delta 8.69(\mathrm{~d}, J=5.4 \mathrm{~Hz}, 2 \mathrm{H}), 7.38(\mathrm{dd}, J=4.5,1.5 \mathrm{~Hz}, 2 \mathrm{H}), 3.65(\mathrm{t}, J=$ $7.0 \mathrm{~Hz}, 2 \mathrm{H}), 3.38(\mathrm{t}, J=6.6 \mathrm{~Hz}, 2 \mathrm{H}), 2.01-1.95(\mathrm{~m}, 2 \mathrm{H}), 1.93-1.88(\mathrm{~m}, 2 \mathrm{H})$. 
naphthalen-2-yl(pyrrolidin-1-yl)methanone<smiles>O=C(c1ccc2ccccc2c1)N1CCCC1</smiles>

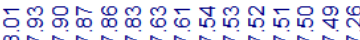

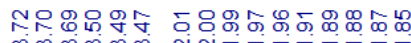

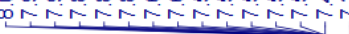

लुखिल

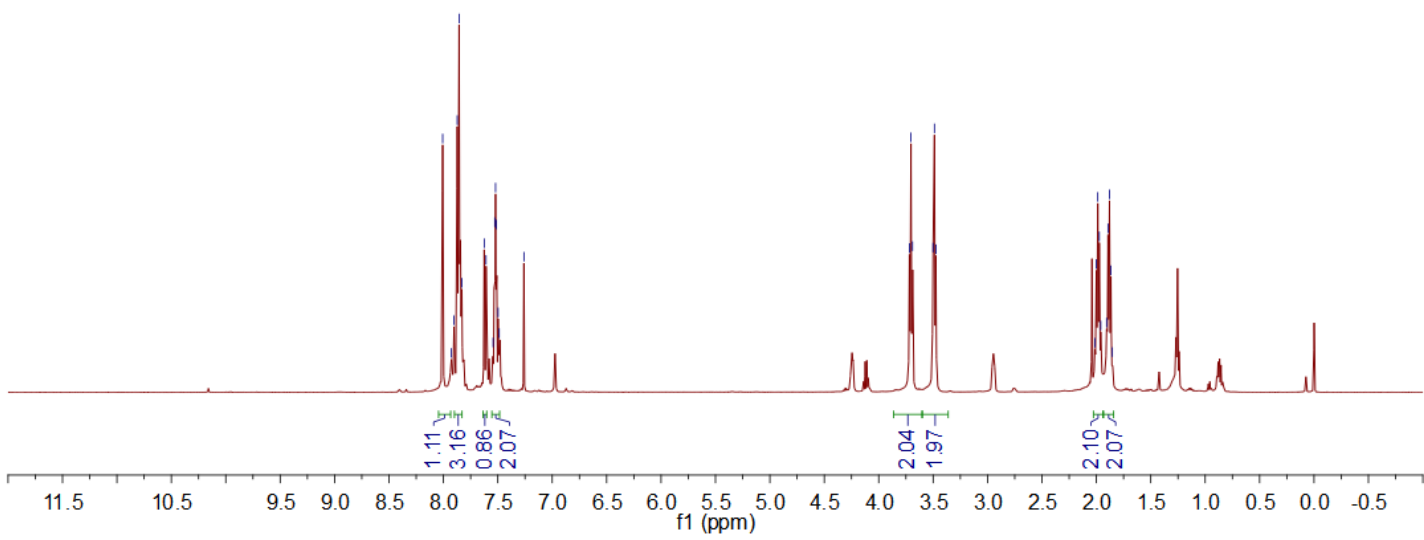

${ }^{1} \mathrm{H} \mathrm{NMR}\left(500 \mathrm{MHz}, \mathrm{CDCl}_{3}\right) \delta 8.01(\mathrm{~s}, 1 \mathrm{H}), 7.96-7.81(\mathrm{~m}, 3 \mathrm{H}), 7.62(\mathrm{~d}, J=8.4 \mathrm{~Hz}, 1 \mathrm{H}), 7.56-$ $7.48(\mathrm{~m}, 2 \mathrm{H}), 3.70(\mathrm{t}, J=7.0 \mathrm{~Hz}, 2 \mathrm{H}), 3.49(\mathrm{t}, J=6.6 \mathrm{~Hz}, 2 \mathrm{H}), 2.03-1.94(\mathrm{~m}, 2 \mathrm{H}), 1.94-1.84$ $(\mathrm{m}, 2 \mathrm{H})$. 
azepan-1-yl(4-nitrophenyl)methanone<smiles>O=C(c1ccc([N+](=O)[O-])cc1)N1CCCCCC1</smiles>

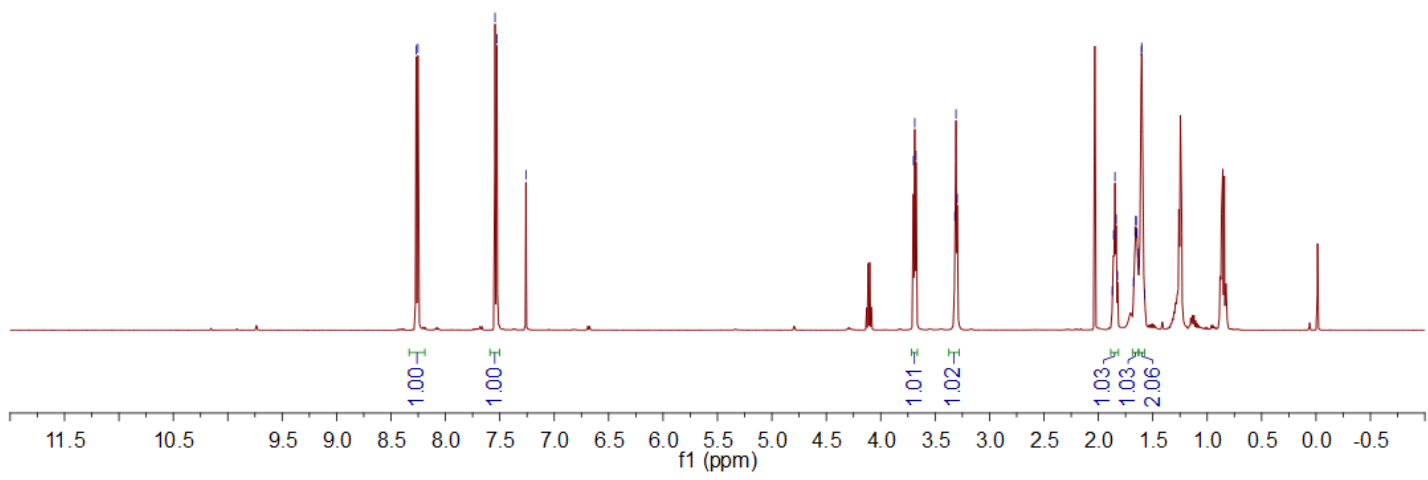

${ }^{1} \mathrm{H}$ NMR $\left(500 \mathrm{MHz}, \mathrm{CDCl}_{3}\right) \delta 8.26(\mathrm{~d}, J=8.5 \mathrm{~Hz}, 2 \mathrm{H}), 7.54(\mathrm{~d}, J=8.5 \mathrm{~Hz}, 2 \mathrm{H}), 3.68(\mathrm{t}, J=6 \mathrm{~Hz}$, $2 \mathrm{H}), 3.31(\mathrm{t}, J=5.5 \mathrm{~Hz}, 2 \mathrm{H}), 1.89-1.81(\mathrm{~m}, 2 \mathrm{H}), 1.65-1.68(\mathrm{~m}, 2 \mathrm{H}), 1.63-1.57(\mathrm{~m}, 4 \mathrm{H})$. 
morpholino(4-nitrophenyl)methanone<smiles>O=C(c1ccc([N+](=O)[O-])cc1)N1CCOCC1</smiles>

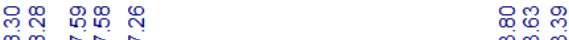

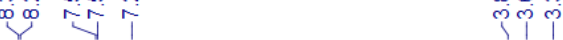

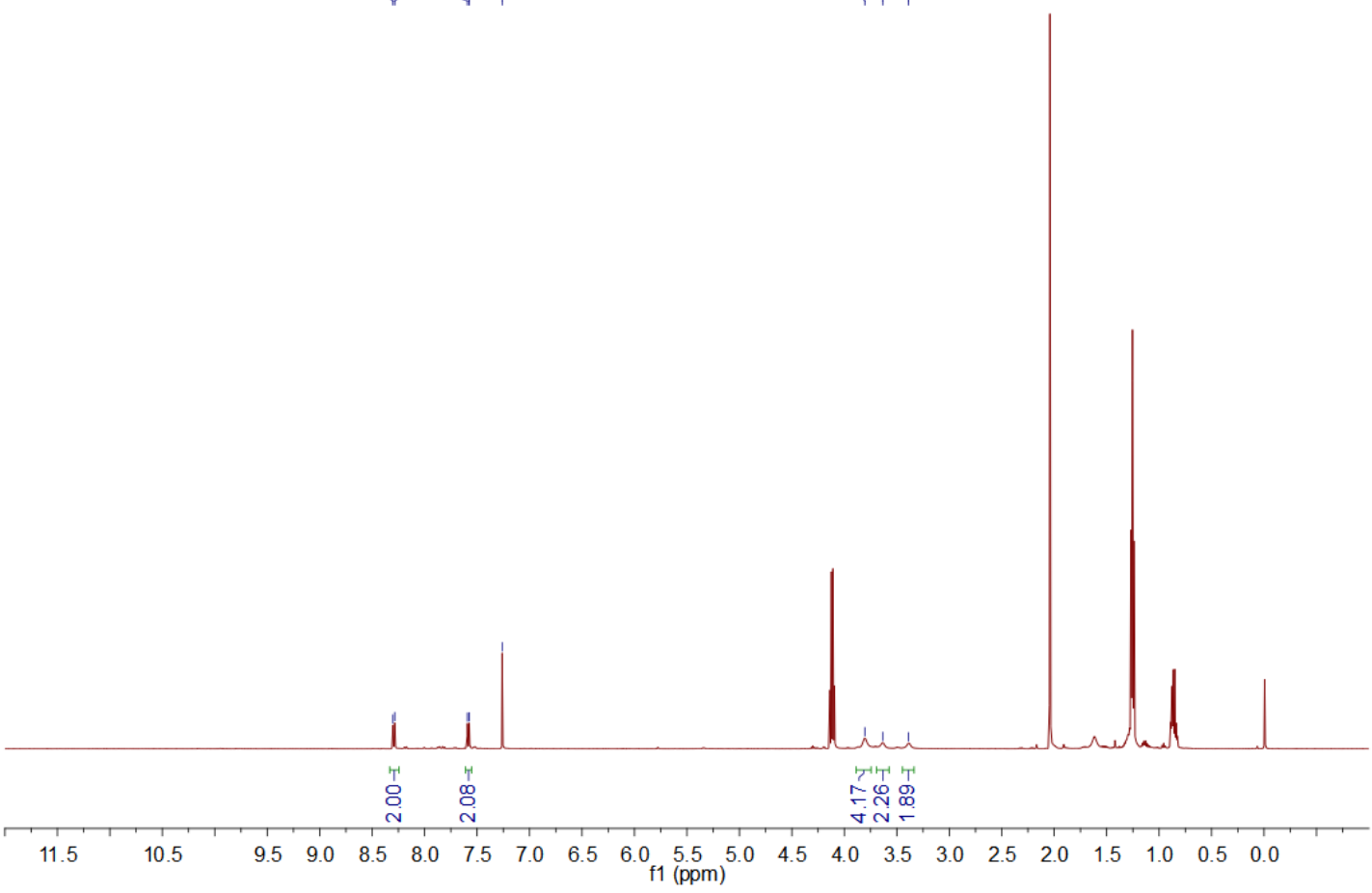

${ }^{1} \mathrm{H}$ NMR $\left(500 \mathrm{MHz}, \mathrm{CDCl}_{3}\right) \delta 8.29(\mathrm{~d}, J=8.6 \mathrm{~Hz}, 2 \mathrm{H}), 7.58(\mathrm{~d}, J=8.6 \mathrm{~Hz}, 2 \mathrm{H}), 3.80(\mathrm{br}, 4 \mathrm{H})$, 3.63 (br, 2H), 3.39 (br, 2H). 
(4-methylpiperazin-1-yl)(4-nitrophenyl)methanone<smiles>CN1CCN(C(=O)c2ccc([N+](=O)[O-])cc2)CC1</smiles>

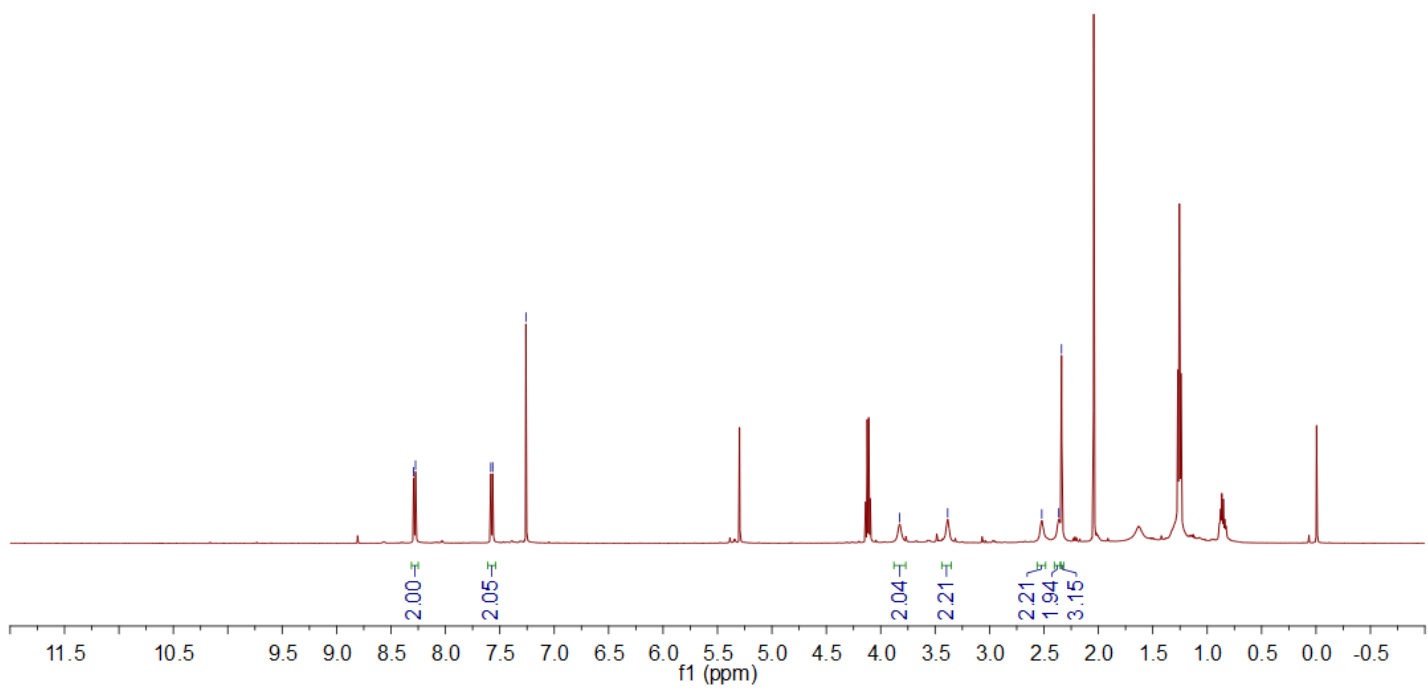

${ }^{1} \mathrm{H}$ NMR $\left(500 \mathrm{MHz}, \mathrm{CDCl}_{3}\right) \delta 8.28(\mathrm{~d}, J=8.7 \mathrm{~Hz}, 2 \mathrm{H}), 7.57(\mathrm{~d}, J=8.7 \mathrm{~Hz}, 2 \mathrm{H}), 3.83(\mathrm{br}, 2 \mathrm{H})$, 3.38 (br, 2H), 2.52 (br, 2H), 2.36 (br, 2H), 2.34 (s, 3H). 


\section{References}

1. Hervé, G.; Tézé, A. Study of $\alpha$ - and $\beta$-Enneatungstosilicates and -Germinates. Inorg. Chem. 1977, 168, 2115-2117.

2. Li, G. B.; Liu, J. M.; Yu, Z. Q.; Wang, W.; Su, C. Y. Assembly of a 1D Coordination Polymer Through in situ Formation of a New Ligand by Double $\mathrm{C}-\mathrm{C}$ Coupling on $\mathrm{CHCl}_{3}$ Under Solvothermal Conditions. Inorg. Chem. 2009, 48, 8659-8661.

3. Liang, H. Q.; Guo, Y.; Shi, Y. S.; Peng, X. S.; Liang, B.; Chen, B. L. A Light-Responsive Metal-Organic Framework Hybrid Membrane with High on/off Photoswitchable Proton Conductivity. Angew. Chem. Int. Ed. 2020, 59, 7732-7737.

4. Li, Z.; Lin, L. D.; Yu, H.; Li, X. X.; Zheng, S. T. All-Inorganic Ionic Porous Material Based on Giant Spherical Polyoxometalates Containing Core-Shell $\mathrm{K}_{6} @ \mathrm{~K}_{36}$-Water Cage. Angew. Chem. Int. Ed. 2018, 48, 15777-15781.

5. Wang, H. N.; Meng, X.; Dong, L. Z.; Chen, Y. F.; Li, S. L.; Lan, Y. Q. Coordination Polymer-Based Conductive Materials: Ionic Conductivity vs. Electronic Conductivity. J. Mater. Chem. A 2019, 7, 24059-24091. 